\title{
Further studies on the gain properties of a Gas Electron Multiplier with a Micro-Induction Gap Amplifying Structure (GEM-MIGAS) aimed at low-energy X-ray detection
}

\author{
J.A. Mir ${ }^{a}, *$ R. Stephenson ${ }^{a}$, N.J. Rhodes ${ }^{\mathrm{a}}$, E.M. Schooneveld ${ }^{\mathrm{a}}$, H. Natal da Luz ${ }^{\mathrm{b}, \mathrm{c}}$, \\ J.F.C.A. Veloso ${ }^{\text {c }}$ J.M.F. Dos Santos ${ }^{\text {c }}$, C.D.R. Azevedo ${ }^{\mathrm{b}, \mathrm{c}}$ \\ ${ }^{a}$ Science and Technology Facilities Council, Rutherford Appleton Laboratory, Chilton, Didcot, Oxon, OX11 0QX, UK \\ ${ }^{\mathrm{b}}$ Departmento de Fisica, Universidade de Coimbra, P-3004-516, Coimbra, Portugal \\ ${ }^{\mathrm{c}}$ Departmento de Física, Universidade de Aveiro, P-3810-193 Aveiro, Portugal
}

Received 22 May 2007; received in revised form 22 June 2007; accepted 4 July 2007

Available online 13 July 2007

\begin{abstract}
A Gas Electron Multiplier with Micro-Induction Gap Amplifying Structure (GEM-MIGAS) is formed when the induction gap of the GEM is set between 50 and $100 \mu \mathrm{m}$ using kapton pillars spaced at regular intervals. This configuration combines the properties of a GEM and Micromegas, allowing operation in tandem to generate high charge gains. We measured the essential operational parameters of this system using argon-isobutane (IB) and helium-IB gas mixtures. The present short induction gap GEM was able to achieve effective gains exceeding $2 \times 10^{4}$ using argon-IB and $10^{5}$ using helium-IB mixtures. In view of the high gains achieved, particularly when using heliumbased gas mixtures, these studies confirmed the possibility of using the present system for high-performance sub-keV X-ray detection. (C) 2007 Elsevier B.V. All rights reserved.
\end{abstract}

PACS: $29.40 . \mathrm{Cs} ; 29.40 . \mathrm{Gx} ; 85.60 . \mathrm{Gz}$

Keywords: Gas electron multiplier; GEM; GEM-MIGAS; Effective gain; X-ray energy resolution

\section{Introduction}

Since the advent of the first photo-lithographic micropattern device in 1987 at Institut Laue-Langevin (ILL), the Micro Strip Proportional Counter (MSGC) [1], there has been a vast proliferation of the second-generation micropattern detectors worldwide. These include the Gas Electron Multiplier (GEM), Micromegas, MicroGap, Microwell and Microdot [2-6]. Significant R\&D effort has been applied to optimise these detectors, extending their upper charge gain limits to qualify them for applications ranging from single-photon UV photosensors to low-energy X-ray detection. In the case of MSGC, various researchers have carried out systematic studies on charge gain as a function of anode-cathode dimensions

${ }^{*}$ Corresponding author. Tel.: + 441235446262 ; fax: +441235446863 .

E-mail address: J.A.Mir@stfc.ac.uk (J.A. Mir).
[7-10], while others have advocated cascaded devices such as the double or triple GEMs [11,12] or the Micro-Hole and Strip Plate (MHSP) detector [13]. The Gas Electron Multiplier with a Micro-Induction Gap Amplifying Structure (GEM-MIGAS) presented in a previous study [14] aims to enhance the effective charge gain of a GEM while remaining compact compared with a double or a triple GEM.

The GEM-MIGAS consists of a conventional GEM coupled to a readout anode where the induction gap is defined by typically $50 \mu \mathrm{m}$ tall kapton pillars spaced at $2 \mathrm{~mm}$ intervals. The operation of a GEM-MIGAS is based on electron multiplication in GEM holes as well as in the induction gap, thus combining the amplification properties of a GEM and a Micromegas in a single structure. This results in elevated charge gains, more efficient charge collection and lower operational voltages with fast output pulses. Owing to the existence of higher induction fields, 
GEM-MIGAS also exhibits a better gain stability as shown in the previous study [14] when compared with the conventional GEM operation with induction fields set around the $1 \mathrm{kV} / \mathrm{cm}$ region [15].

In the previous study [14], the effective charge-gain properties of the short induction gap were examined for the GEM voltage range of 400-500 V and induction field range of $0-40 \mathrm{kV} / \mathrm{cm}$ using $\operatorname{Ar}(75 \%)$-isobutane (IB)(25\%). The highest effective gain was found to be approximately 27,000 by setting the voltage across the GEM holes to $500 \mathrm{~V}$ and induction field at $30 \mathrm{kV} / \mathrm{cm}$. In addition, the best $\mathrm{X}$-ray energy resolution at $5.89 \mathrm{keV}$ was found to be $15.2 \%$ FWHM when the voltage across the GEM holes was $470 \mathrm{~V}$ (effective gain 1900) and the induction field was $6 \mathrm{kV} / \mathrm{cm}$. However, it was found that the X-ray energy resolution deteriorated rapidly when the induction fields exceeded $15 \mathrm{kV} / \mathrm{cm}$, probably due to mechanical defects in the readout anode (micromesh).

In the present study, the GEM voltage range was extended to $100-550 \mathrm{~V}$ and induction field range to $0-100 \mathrm{kV} / \mathrm{cm}$ using $\operatorname{Ar}(75 \%)-\operatorname{IB}(25 \%)$. In view of the high charge gain properties of the GEM-MIGAS, we have taken a set of measurements using $\mathrm{He}(75 \%)-\mathrm{IB}(25 \%)$ to exploit the potential of the current device for future synchrotron radiation work involving total electron yield [16] and a micro-pattern-based fluorescence detector for the low $\mathrm{X}$-ray energy region $(270 \mathrm{eV}-1.0 \mathrm{keV})$ [17].

\section{Method}

The experimental arrangement of the present system is shown in Fig. 1. The X-ray-sensitive area of the present detection system consisted of a $10 \mathrm{~mm} \times 10 \mathrm{~mm}$ GEM supported on a G-10 frame. The GEM was fabricated at the CERN TS-DEM workshop and consisted of a $50 \mu \mathrm{m}$ thick copper clad $(5 \mu \mathrm{m})$ kapton foil with $70(50) \mu \mathrm{m}$ holes patterned at $140 \mu \mathrm{m}$ hole pitch. The induction electrode is a micromesh, also manufactured at CERN TS-DEM workshop, and consisted of a $5 \mu \mathrm{m}$ thick copper mesh with $25 \mu \mathrm{m}$ holes etched at a pitch of $50 \mu \mathrm{m}$ with $50 \mu \mathrm{m}$ tall and $150 \mu \mathrm{m}$ diameter kapton pillars distributed at $2 \mathrm{~mm}$ intervals. In order to construct the GEM-MIGAS, the micromesh was glued on to a readout board with $10 \mathrm{~mm} \times 10 \mathrm{~mm}$ anode and held together with the GEM frame. The drift depth was set at $38 \mathrm{~mm}$, whereby three $10 \mathrm{~mm}$ thick aluminium concentric rings, each separated

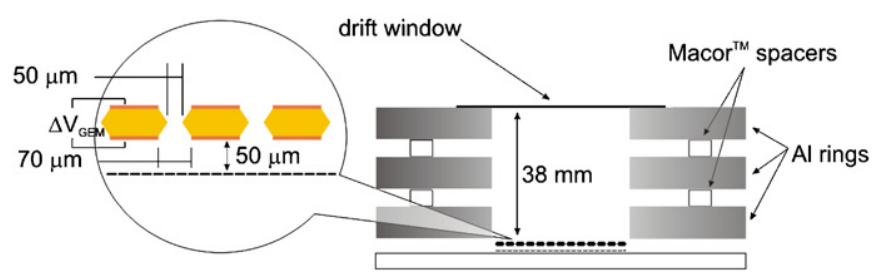

Fig. 1. Schematic diagram of the experimental system used for studying the Gas Electron Multiplier with a Micro-Induction Gap Amplifying Structure (GEM-MIGAS). from each other by $3 \mathrm{~mm}$, were used to achieve a graded drift potential. The topmost ring was glued with $100 \mu \mathrm{m}$ thick aluminium foil to define the drift window.

In the experimental studies described in the following sections, a Mn-K X-ray (5.89 keV) beam illuminated the detector drift space perpendicular to the GEM and micromesh planes. In this study, the detection chamber was operated at a constant gas flow rate where the gas composition was controlled by Brooks mass-flow controllers (model 5850E) built into a rig that was constructed using stainless steel tubing. $\operatorname{An} \operatorname{Ar}(75 \%)-\operatorname{IB}(25 \%)$ or $\mathrm{He}(75 \%)-\mathrm{IB}(25 \%)$ gas mixture was used throughout this study. The count rates used throughout these studies were kept in the region of $10 \mathrm{kHz}$.

The drift electrode and the GEM mesh were operated negative with respect to the micromesh that was held close to the earth. The micromesh was connected electrically to an Ortec preamplifier (model 142A). The preamplifier output was then fed into an Ortec shaping amplifier (model $575 \mathrm{~A}$ ) with shaping time constants adjusted to $0.5 \mu$ s. The bipolar output of the shaping amplifier was in turn fed into an Ortec pulse height analyser. The effective gain and the $\mathrm{X}$-ray energy resolution were examined as a function of the induction field, $E_{\mathrm{I}}$, and the voltage differences applied across the GEM holes, $\Delta V_{\mathrm{GEM}}$. The drift field, $E_{\mathrm{d}}$, was maintained at approximately $0.25 \mathrm{kV} / \mathrm{cm}$ throughout these studies. In each set of measurements, either $E_{\mathrm{I}}$ or $\Delta V_{\mathrm{GEM}}$ was gradually increased up to the onset of microdischarges (i.e. no observable discharges during the time of the pulse height acquisition in typically $300 \mathrm{~s}$ duration). The microdischarge processes occur when the upper voltage hold-off capability of kapton or the gas being used is exceeded and are accompanied by a surge in current within the GEM holes or in the induction gap. In order to protect GEMMIGAS, we limited our currents to $5 \mu \mathrm{A}$, beyond which the high-voltage power supplies were automatically turned off.

\section{Effective gain and X-ray energy resolution}

The effective gain was calculated by determining the total charge for a given set of voltage settings. This was achieved by generating a known voltage pulse into an accurately known capacitor located at the preamplifier test input and correlating it with pulse height spectrum (centroid) from $5.89 \mathrm{keV}$ X-rays. The effective gain was then derived by dividing the charge at the centroid of the pulse height distribution with the number of primary electrons, $N_{0}$, for a given gas mixture. This required the knowledge of the mean energy, $w$, required to create an electron-ion pair during the initial interaction between the $5.89 \mathrm{keV}$ photon and the counter gas molecule $[18,19]$. The parameter $w$ for $\operatorname{Ar}(75 \%)-\operatorname{IB}(25 \%)$ and $\mathrm{He}(75 \%)-$ $\mathrm{IB}(25 \%)$ was assumed to be 25.25 and $36.5 \mathrm{eV}$, respectively.

Fig. 2 shows the variation of the effective gain as a function of the voltage applied across the GEM holes with induction field set at $6 \mathrm{kV} / \mathrm{cm}$ (i.e. no charge amplification 


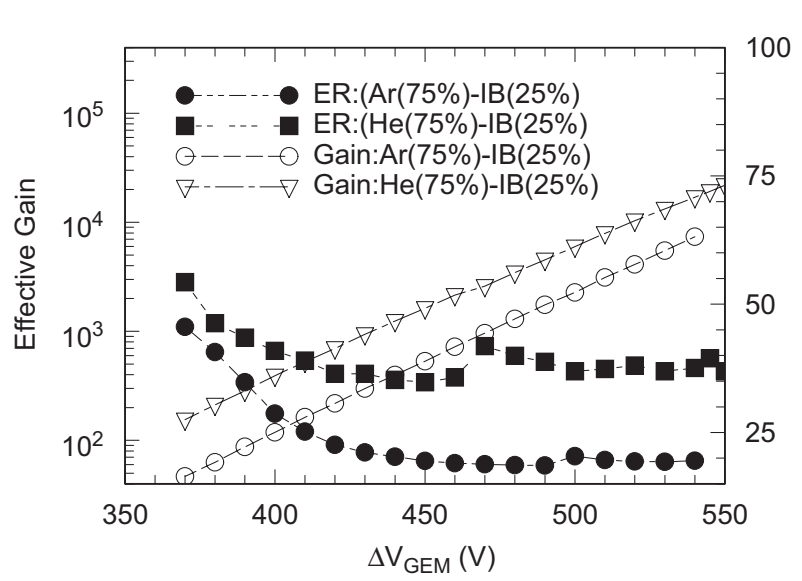

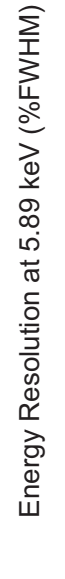

Fig. 2. Effective gain and X-ray energy resolution at $5.89 \mathrm{keV}$ as a function of the voltage applied across the GEM holes, $\Delta V_{\mathrm{GEM}}$, using $\operatorname{Ar}(75 \%)$-isobutane $(25 \%)$ and $\mathrm{He}(75 \%)$-isobutane $(25 \%)$. The induction field $E_{\mathrm{I}}$ was set at $6 \mathrm{kV} / \mathrm{cm}$ and drift field $E_{\mathrm{d}}$ at $0.25 \mathrm{kV} / \mathrm{cm}$.

in the induction gap) for $\operatorname{Ar}(75 \%)-\operatorname{IB}(25 \%)$ and of $\mathrm{He}(75 \%)-\mathrm{IB}(25 \%)$. In the case of $\operatorname{Ar}(75 \%)-\mathrm{IB}(25 \%)$, the maximum achievable gain was found to be approximately $7.4 \times 10^{3}$, compared with a gain of $7.0 \times 10^{3}$ in another study [15] using a single GEM with $\operatorname{Ar}(70 \%)-\mathrm{CO}_{2}(30 \%)$ with $\Delta V_{\mathrm{GEM}}$ at $570 \mathrm{~V}$ and $E_{\mathrm{I}}$ at $5 \mathrm{KV} / \mathrm{cm}$. The main feature shown in Fig. 2 is a four-fold increase in the effective gain when using helium-based gas mixture and is consistent with the observations reported by others [20,21].

The errors associated with the measurement of the effective gains in this work were approximately $\pm 10 \%$. The main sources of the errors were attributed to charge calibration and effective gain shifts due to ambient variations (pressure $P$ and temperature $T$ ). Since the stray capacitance at the test input stage of the Ortec preamplifier is not known, we could obtain $\pm 5 \%$ charge calibration accuracy by cross-calibrating the test input capacitance with an additional accurately known test capacitance $( \pm 50 \mathrm{fF})$ placed at the preamplifier input. The effective gain of flow-type gaseous detectors is effected by the ratio of ambient variables $P$ and $T$ (Ref. [8] and references therein), where $P$ is the gas pressure in mbar and $T$ is its absolute temperature in K. Fig. 3 shows the effective gain of the present GEM over a 1-month period, plotted as a function $q$ where $q$ is the ratio $P / T$. Fitting an exponential curve to the data gives the effective gain sensitivity of the detector to changes in the ambient conditions. In the present case, this evaluates to $1.55 \mathrm{~K} / \mathrm{mbar}$ as shown in Fig. 3 and implies, for example, an effective gain shift of $1.9 \%$ if $P$ remained constant (1000 mbar) and $T$ changed $\pm 1{ }^{\circ} \mathrm{C}$ from $20^{\circ} \mathrm{C}$. The standard deviation for the average effective gain of 1270 in Fig. 3 is 39.5, giving a fractional error of $3.1 \%$.

Fig. 2 also shows the variation of the X-ray energy resolution at $5.89 \mathrm{keV}$ as a function of the voltage applied across the GEM holes with the induction field at $6 \mathrm{kV} / \mathrm{cm}$. The optimum X-ray energy resolution occurred when the effective gain exceeded 1000 . The optimum energy resolu-

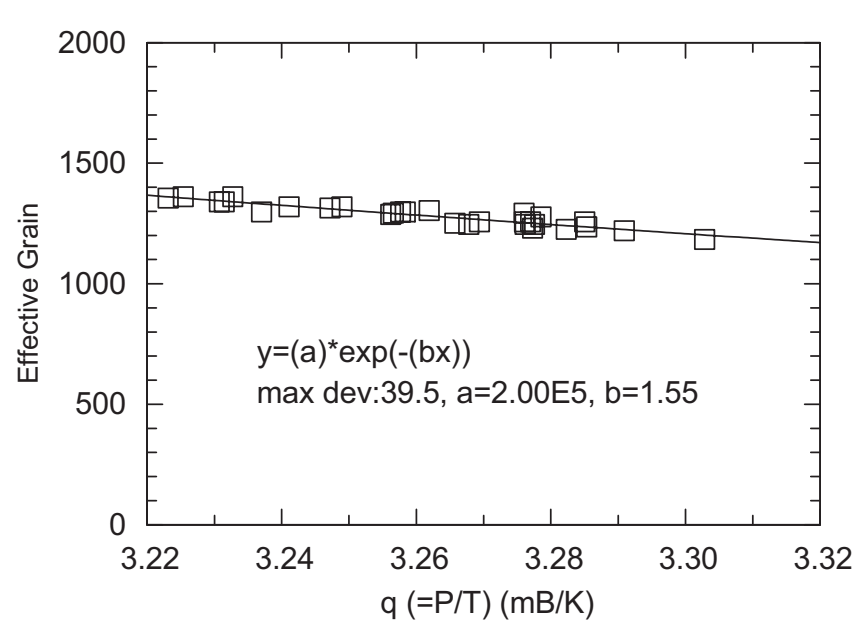

Fig. 3. The effective gain measured over a period of 1 month using $\operatorname{Ar}(75 \%)$-isobutane $(25 \%)$ as a function of the ambient parameter $P / T$ at a constant $\Delta V_{\mathrm{GEM}}$ and $E_{\mathrm{I}}$. The experimental data were fitted with $y=(a) \times \exp (-(b x))$, yielding a standard deviation of 39.5 for the average effective gain of 1270 . Effective gain sensitivity with respect to $P / T$ was found to be $1.55 \mathrm{~K} / \mathrm{mbar}$.

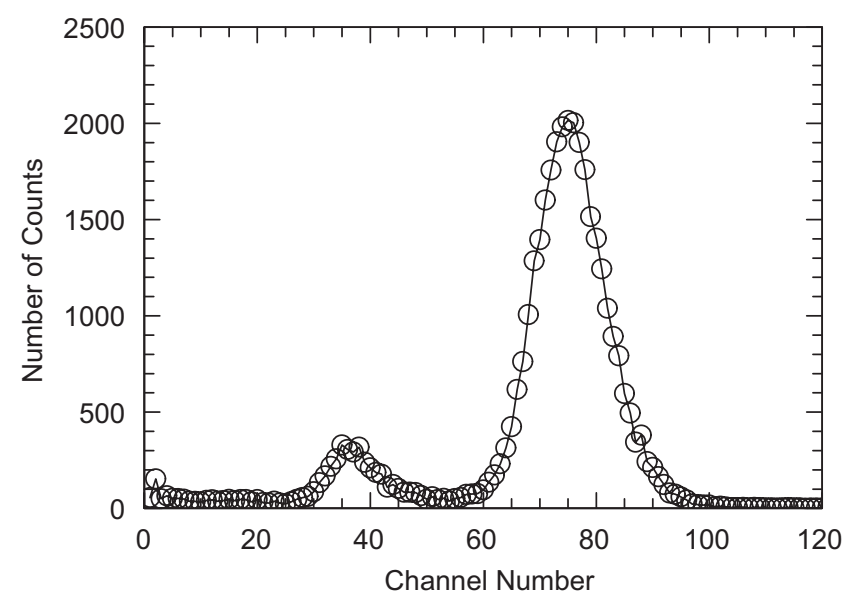

Fig. 4. Pulse height spectrum for $5.89 \mathrm{keV} \mathrm{X}$-rays using the GEM at an effective gain of $7.4 \times 10^{3}$ in $\operatorname{Ar}(75 \%)$-isobutane $(25 \%)$ with $\Delta V_{\mathrm{GEM}}=$ $550 \mathrm{~V}, E_{\mathrm{I}}=6 \mathrm{kV} / \mathrm{cm}$ and $E_{\mathrm{d}}=0.25 \mathrm{kV} / \mathrm{cm}$.

tion for $\operatorname{Ar}(75 \%)-\mathrm{IB}(25 \%)$ and $\mathrm{He}(75 \%)-\mathrm{IB}(25 \%)$ was found to be approximately $18 \%$ and $35 \%$, FWHM, respectively. The error associated with measuring the $\mathrm{X}$-ray energy resolution was within $\pm 2 \%$.

The pulse height distribution at $5.89 \mathrm{keV}$ using $\operatorname{Ar}(75 \%)-\mathrm{IB}(25 \%)$ with $\Delta V_{\mathrm{GEM}}=550 \mathrm{~V}$, gain $=7.4 \times 10^{3}$ is shown in Fig. 4. In addition, the pulse height distribution for $\mathrm{He}(75 \%)-\mathrm{IB}(25 \%)$ with $\Delta V_{\mathrm{GEM}}=540 \mathrm{~V}$, gain $=$ $2.2 \times 10^{3}$ is shown in Fig. 5, which also shows an aluminium-K fluorescence peak $(1.5 \mathrm{keV})$ caused by photon interactions with the chamber walls.

Fig. 6 shows the variation of the effective gain using $\operatorname{Ar}(75 \%)-\operatorname{IB}(25 \%)$ as a function of the induction field for the range $E_{\mathrm{I}}=0.4-100 \mathrm{kV} / \mathrm{cm}$ for a number of different voltages across the GEM holes $\left(\Delta V_{\mathrm{GEM}}=100,200,300\right.$, 


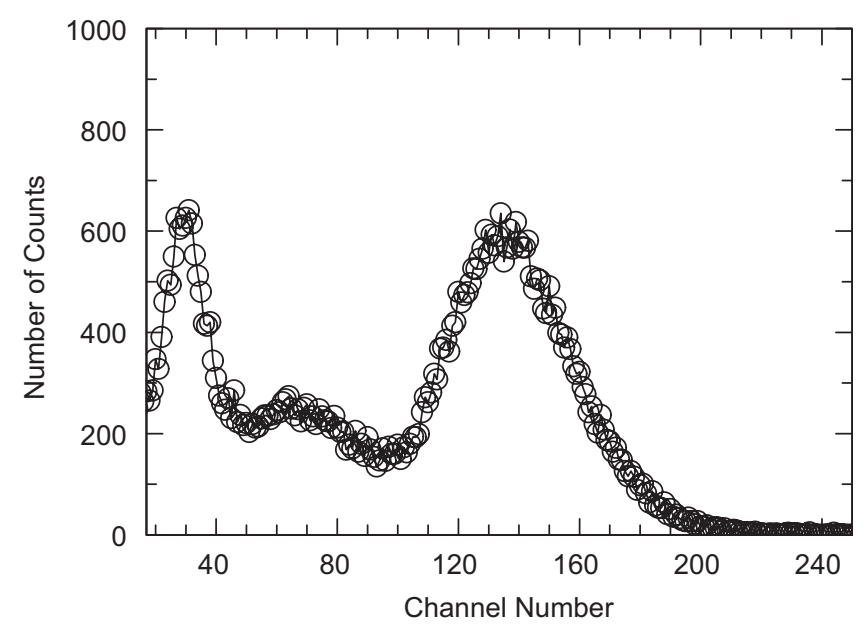

Fig. 5. Pulse height spectrum for $5.89 \mathrm{keV}$ X-rays using the GEM at an effective gain of $2.2 \times 10^{3}$ in $\mathrm{He}(75 \%)$-isobutane $(25 \%)$ with $\Delta V_{\mathrm{GEM}}=$ $540 \mathrm{~V}, E_{\mathrm{I}}=6 \mathrm{kV} / \mathrm{cm}$ and $E_{\mathrm{d}}=0.25 \mathrm{kV} / \mathrm{cm}$.

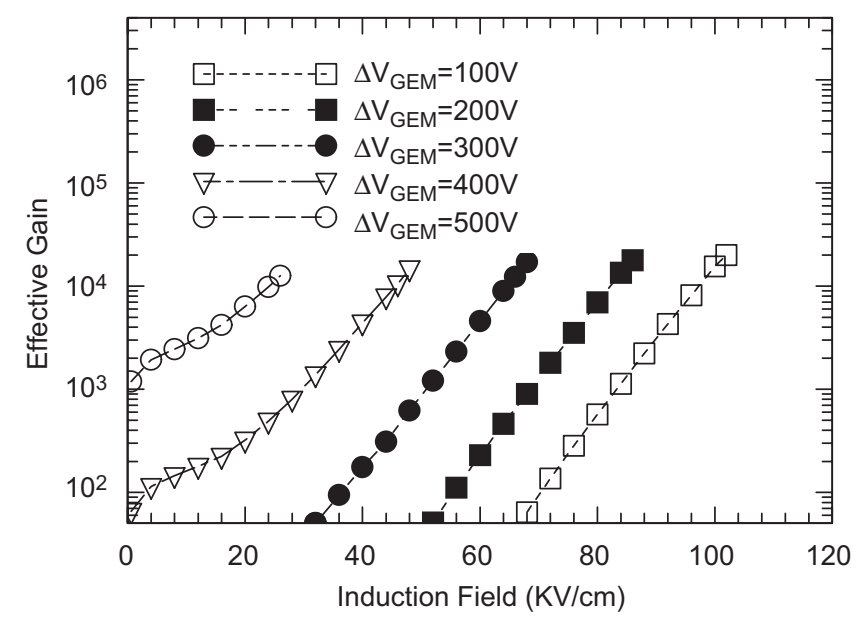

Fig. 6. Effective gain as a function of the induction field using $\operatorname{Ar}(75 \%)$-isobutane $(25 \%)$ for $\Delta V_{\mathrm{GEM}}=100,200,300,400$ and $500 \mathrm{~V}$. In all cases $E_{\mathrm{d}}=0.25 \mathrm{kV} / \mathrm{cm}$.

400 and $500 \mathrm{~V}$ ). As shown in Fig. 6, a slightly higher effective gain before microdischarges occurred with decreasing values of $\Delta V_{\mathrm{GEM}}$. It is worth noting that, in the previous study concerning the maximum achievable gain using the GEM-MIGAS, a typical value was found to be approximately $2 \times 10^{4}$. By extending the upper induction field to $100 \mathrm{kV} / \mathrm{cm}$ in the present study, no further gain enhancement was observed for the case of the argon-based gas mixture. The present results using an argon-based mixture are comparable to another study [22], albeit using $\operatorname{Ar}(70 \%)-\mathrm{CO}_{2}(30 \%)$, with the combined operation of a GEM and micromegas where the maximum gain in the region of $2 \times 10^{4}$ was realised $\left(\Delta V_{\mathrm{GEM}} \sim 400 \mathrm{~V}\right.$ and $\left.E_{\mathrm{I}} \sim 80 \mathrm{kV} / \mathrm{cm}\right)$.

Fig. 7 shows the variation of the X-ray energy resolution as a function of induction field using $\operatorname{Ar}(75 \%)-\operatorname{IB}(25 \%)$

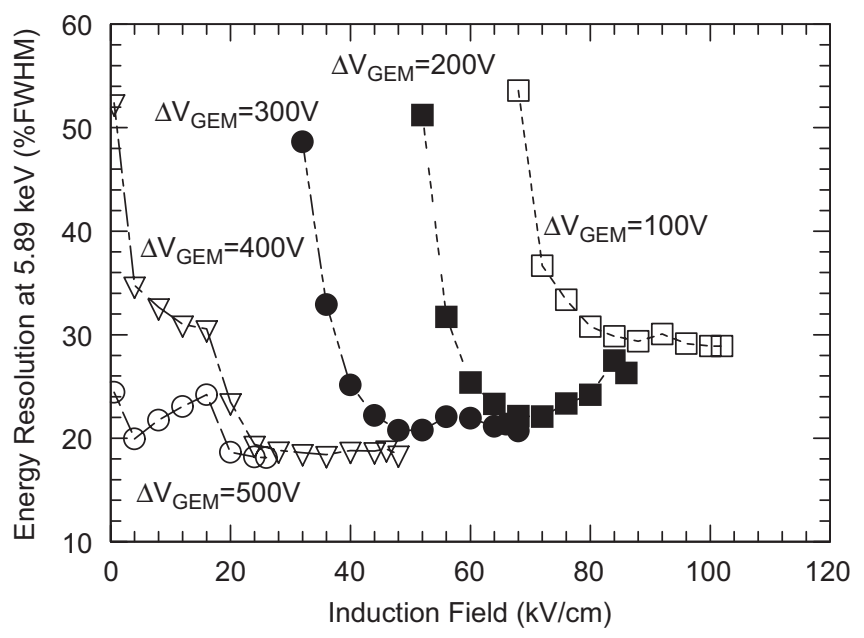

Fig. 7. X-ray energy resolution ( $\%$ FWHM) of the $\mathrm{Mn}-\mathrm{K}$ X-rays $(5.89 \mathrm{keV})$ as a function of the induction field using $\operatorname{Ar}(75 \%)$-isobutane $(25 \%)$ for $\Delta V_{\mathrm{GEM}}=100,200,300,400$ and $500 \mathrm{~V}$. In all cases $E_{\mathrm{d}}=0.25 \mathrm{kV} / \mathrm{cm}$.

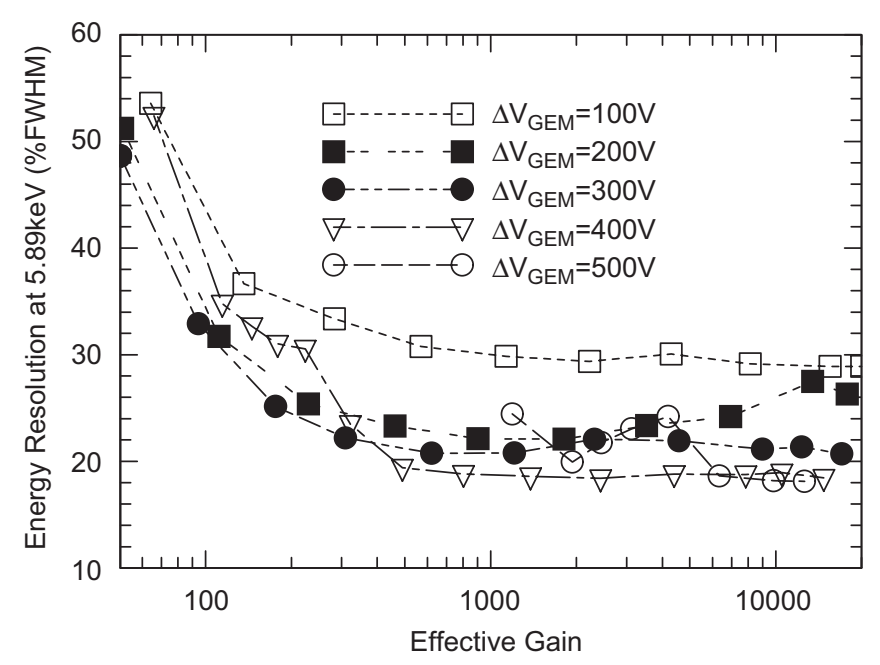

Fig. 8. X-ray energy resolution $(\% \mathrm{FWHM})$ of the $\mathrm{Mn}-\mathrm{K}$ X-rays $(5.89 \mathrm{keV})$ as a function of the effective charge gain using $\operatorname{Ar}(75 \%)$ isobutane $(25 \%)$ for $\Delta V_{\mathrm{GEM}}=100,200,300,400$ and $500 \mathrm{~V}$. In all cases $E_{\mathrm{d}}=0.25 \mathrm{kV} / \mathrm{cm}$.

for a number of different voltages across the GEM holes. In addition, Fig. 8 shows the energy resolution as a function of the effective gain. The optimum resolution for each curve occurred when the effective gain exceeded values above 1000. However, the best X-ray energy resolution was found to be approximately $18 \% \mathrm{FWHM}$ when $\Delta V_{\mathrm{GEM}}$ was set between 400 and $500 \mathrm{~V}$. The energy resolution for a given $\Delta V_{\mathrm{GEM}}$ remained constant up the maximum usable $E_{\mathrm{I}}$. This is in contrast to the previous study [14], where resolution degraded rapidly above $E_{\mathrm{I}}=15 \mathrm{kV} / \mathrm{cm}$. We attribute this difference to mechanical defects/imperfections in the readout anode (micromesh) during the construction of the first prototype.

Fig. 9 shows the variation of the effective gain using $\mathrm{He}(75 \%)-\mathrm{IB}(25 \%)$ as a function of the induction field for 


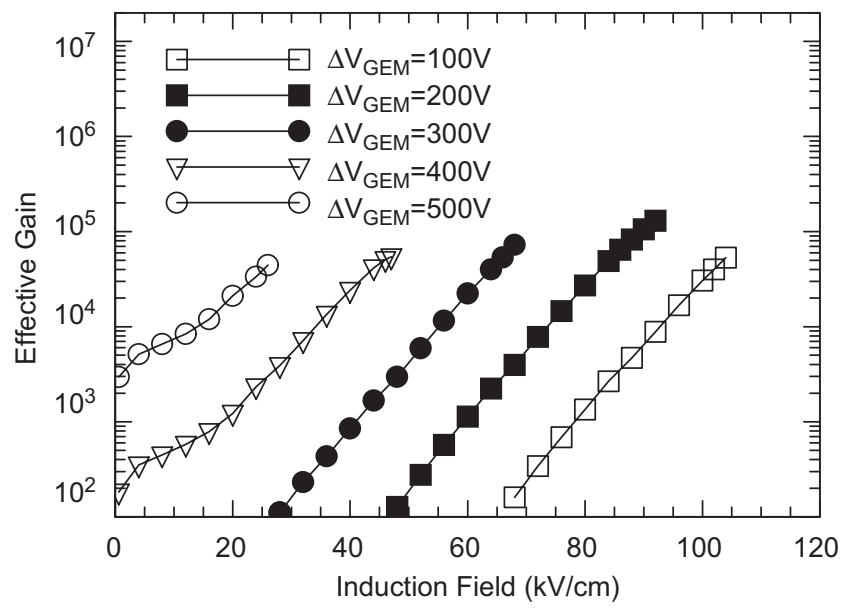

Fig. 9. Effective gain as a function of the induction field using $\mathrm{He}(75 \%)$-isobutane $(25 \%)$ for $\Delta V_{\mathrm{GEM}}=100,200,300,400$ and $500 \mathrm{~V}$. In all cases $E_{\mathrm{d}}=0.25 \mathrm{kV} / \mathrm{cm}$.

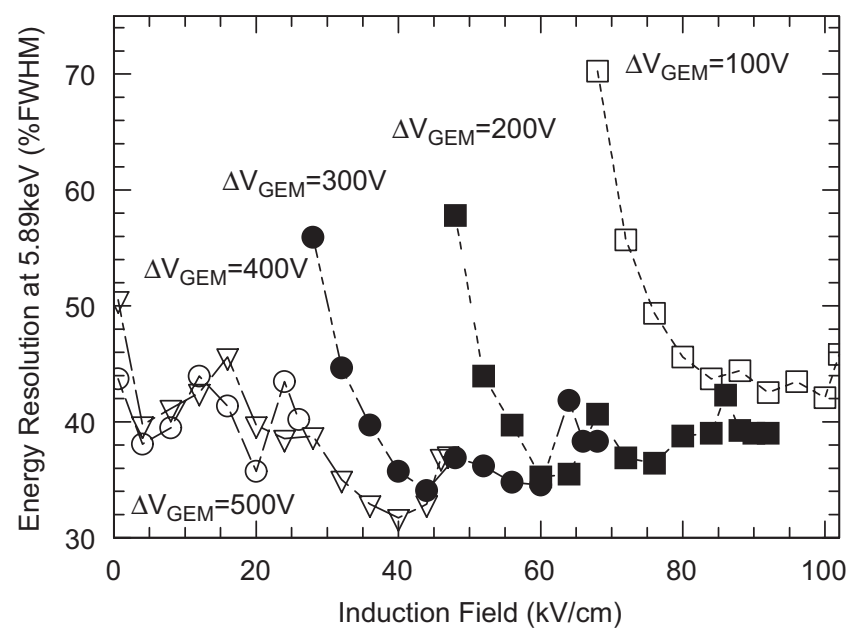

Fig. 10. X-ray energy resolution $(\% \mathrm{FWHM})$ of the $\mathrm{Mn}-\mathrm{K}$ X-rays $(5.89 \mathrm{keV})$ as a function of the induction field using $\mathrm{He}(75 \%)$-isobu$\operatorname{tane}(25 \%)$ for $\Delta V_{\mathrm{GEM}}=100,200,300,400$ and $500 \mathrm{~V}$. In all cases $E_{\mathrm{d}}=0.25 \mathrm{kV} / \mathrm{cm}$.

the range $E_{\mathrm{I}}=0.4-100 \mathrm{kV} / \mathrm{cm}$ for a number of different voltages across the GEM holes $\left(\Delta V_{\mathrm{GEM}}=100,200,300\right.$, 400 and $500 \mathrm{~V}$ ). Extending the upper induction field to $100 \mathrm{kV} / \mathrm{cm}$ in the case of the helium-IB mixture, a significant gain enhancement was observed. The highest gain before the onset of instabilities here was found to be $1.3 \times 10^{5}$. We note that the highest effective charge gains are obtained when $\Delta V_{\mathrm{GEM}}$ is set between 100 and $300 \mathrm{~V}$ with $E_{\mathrm{I}}$ at $75-100 \mathrm{kV} / \mathrm{cm}$. The achieved gains are sufficiently high to allow the use of the GEM-MIGAS as a single-electron counting device (or single-photon counting device, when coupled to a suitable photosensor, e.g. a CsI film coating the GEM upper surface).

Fig. 10 shows the variation of the X-ray energy resolution as a function of induction field using $\mathrm{He}(75 \%)-\mathrm{IB}(25 \%)$ for a number of different voltages across the GEM holes. In addition, Fig. 11 shows the

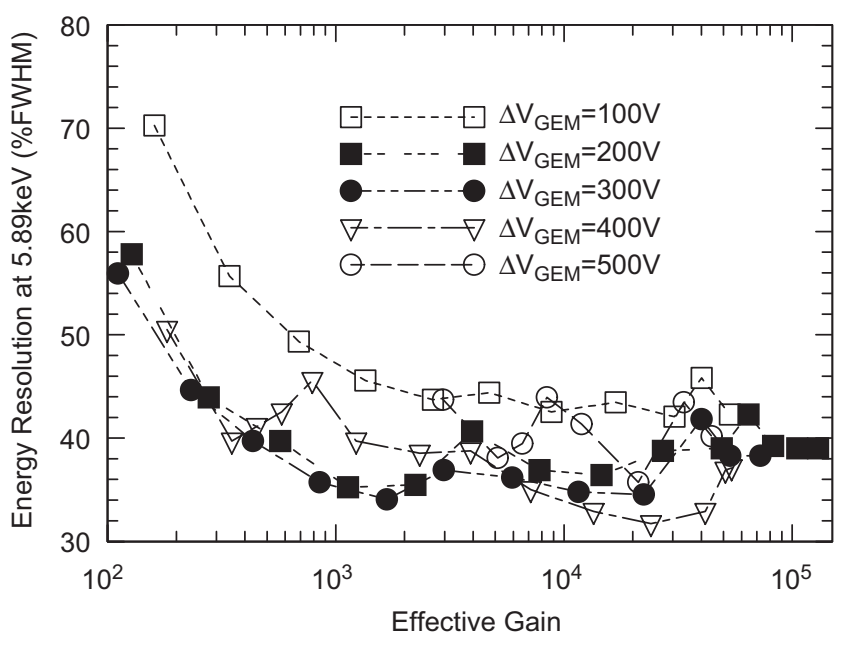

Fig. 11. X-ray energy resolution (\%FWHM) of the $\mathrm{Mn}-\mathrm{K}$ X-rays $(5.89 \mathrm{keV})$ as a function of the effective charge gain using $\mathrm{He}(75 \%)$-isobutane $(25 \%)$ for $\Delta V_{\mathrm{GEM}}=100,200,300,400$ and $500 \mathrm{~V}$. In all cases $E_{\mathrm{d}}=0.25 \mathrm{kV} / \mathrm{cm}$

energy resolution as a function of the effective gain. The optimum resolution for each curve occurred when the effective gain exceeded values above 1000 . However, the best X-ray energy resolution was found to be approximately $32 \%$ FWHM when $\Delta V_{\text {GEM }}$ was set between 300 and $500 \mathrm{~V}$.

\section{Conclusion}

The effective gain and the X-ray energy resolution of the GEM-MIGAS were investigated for a wider range of voltage across the GEM holes and induction gap than reported previously [14]. In this study, the gas mixtures consisting of $\operatorname{Ar}(75 \%)-\mathrm{IB}(25 \%)$ and $\mathrm{He}(75 \%)-\mathrm{IB}(25 \%)$ were used. For the argon-based gas mixture, the highest effective gain was found to be approximately $2 \times 10^{4}$. However, much higher effective gains were observed when using $\mathrm{He}(75 \%)-\mathrm{IB}(25 \%)$, where effective gains in the region of 100,000 were achieved. In general, the highest effective gains were obtained when using lower voltage across the GEM holes (100-300 V) and highest induction fields. Conversely, the X-ray energy resolution improved by using higher voltage across the GEM holes $(300-500 \mathrm{~V})$.

In view of the high effective gains obtained with the conventional GEM operation in helium-isobutane gas mixture and more so with the GEM-MIGAS configuration, we are now pursuing low-energy fluorescent X-ray detection from industrial samples. This work is being carried out at the Science and Technology Facilities Council, Daresbury Laboratory, UK, and will be presented elsewhere at a future date [17].

\section{Acknowledgments}

We acknowledge financial support from STFC's ISIS Gas Detector Development Programme as well as 
assistance from Project POCI/FP/81948/2007 through FEDER and FCT (Lisbon).

\section{References}

[1] A. Oed, Nucl. Instr. and Meth. A 263 (2-3) (1988) 351.

[2] F. Sauli, Nucl. Instr. and Meth. A 386 (1997) 531.

[3] Y. Giomataris, Nucl. Instr. and Meth. A 419 (2-3) (1998) 239.

[4] R. Bvellazzini, et al., Nucl. Instr. and Meth. A 368 (1) (1995) 259.

[5] R. Bellazzini, et al., Nucl. Instr. and Meth. A 423 (1) (1999) 125.

[6] S.F. Biagi, et al., Nucl. Instr. and Meth. A 361 (1-2) (1995) 72.

[7] J.E. Bateman, et al., IEEE Trans. Nucl. Sci. NS-49 (3 Part 2) (2002) 894.

[8] J.E. Bateman et al, IEEE Trans. Nucl. Sci. NS-49 (4 Part 1) (2002) 1644.

[9] J.E. Bateman, et al., Nucl. Instr. and Meth. A 513 (1-2) (2003) 273.

[10] J.J. Florent, et al., The electrostatic field in microstrip chambers and its influence on detector performance, CERN-PPE/92-78, 1992.

[11] F. Sauli, et al., IEEE Trans. Nucl. Sci NS-47 (6 Part 2) (2000) 2070.
[12] F. Sauli, et al., Nucl. Instr. and Meth. A 490 (1-2) (2002) 177.

[13] J.F.C.A. Veloso, et al., Rev. Sci. Instrum. 71 (6) (2000) 2371.

[14] J.A. Mir, et al., Nucl. Instr. Meth. A 573 (2007) 179.

[15] S. Bachmann, et al., Charge amplification and transfer processes in the gas electron multiplier, Technical Report, CERN-EP 99-48, 1999.

[16] T. Rayment, S.L. Schroeder, G.D. Moggridge, J.E. Bateman, G.E. Derbyshire, R. Stephenson, Rev. Sci. Instrum. 71 (10) (2000) 3640.

[17] J.A. Mir, et al., Low X-ray energy fluorescence gas electron multiplier (GEM) for XAS studies, IEEE Trans. Nucl. Sci. (2007) (to be published).

[18] F. Sauli, Principles of operation of multiwire proportional and drift chambers, Technical Report, CERN 77-09, 1977.

[19] A. Sharma, Properties of some gas mixtures used in tracking detectors, ICFA 16 (1998).

[20] G. Charpak, et al., Nucl. Instr. and Meth. A 478 (1-2) (2002) 26.

[21] A. Fornaini, Nucl. Instr. and Meth. A 546 (1-2) (2005) 270.

[22] S. Kane, A study of a combination of a MICROMEGAS + GEM chamber in $\mathrm{A}-\mathrm{CO}_{2}$ gas, Conference Record, IEEE Symposium on Nuclear Science and Medical Imaging, San Diego, 2001. 